\title{
FINITE ELEMENT ANALYSIS OF SWITCHED RELUCTANCE MOTOR WITH ROTOR POSITION BASED CONTROL
}

\author{
${ }^{1}$ Daniel MARCSA, ${ }^{2}$ Miklós KUCZMANN \\ Department of Automation, Széchenyi István University \\ Egyetem tér 1, H-9026 Györ, Hungary \\ e-mail: ${ }^{1}$ marcsa@maxwell.sze.hu, ${ }^{2}$ kuczmann@maxwell.sze.hu
}

Received 9 April 2016; accepted 16 June 2016

\begin{abstract}
This research presents a field-circuit coupled parallel finite element model of a switched reluctance motor embedded in a simple closed loop control system. The parallel numerical model is based on the Schur-complement method coupled with an iterative solver. The used control system is the rotor position based control, which is applied to the FEM model. The results and parallel performance of the voltage driven finite element model are compared with the results from the current driven model. Moreover, the results of the start-up of the loaded motor show why the model accuracy is important in the control loop.
\end{abstract}

Keywords: Coupled finite element method, Switched reluctance motor, Position control, Domain decomposition

\section{Introduction}

The Switched Reluctance Motor (SRM) drives [1], [2] are widely used in special applications like conveyor belts, compressors, and vacuum cleaners because of the advantages like simple construction, no winding on rotor, high speed operation and high temperature handling capability. But its applications are restricted to some areas because of its high ripple content in the torque due to the saliency of the stator and rotor. Because the double saliency structure, necessary for the machine to produce reluctance torque, which complicates the analysis and control of the SRM [1], [2].

Precise control of electric motors is an area of active research worldwide. Efforts concentrate on finding solutions which guarantee high immunity of the drive system to external disturbances, for instance changing load, in terms of realizing prescribed 
trajectories while minimizing associated errors in velocity or torque [1], [3]. However, the appropriate model of the electric motor is very important task of the control. Further, to set up the state space representation of a switched reluctance motor is impossible without any simplifications or experimental data are needed to estimate the unknown parameters of the parametric model of the SRM [1]. Ergo, to create the appropriate model of the SRM without simplifications or measurement, the computational electromagnetics can be an alternative technique.

One of the most popular techniques of computational electromagnetics is the Finite Element Method (FEM) [3], [4], [5]. FEM is a very useful technique to the appropriate modelling of switched reluctance motor. But only the finite element method in itself is not enough for this electro-magneto-mechanical coupled system. Modelling of physical behavior of the electromechanical devices is a very complex task, because electromagnetic field has strong interaction with other physical fields and external circuits [3], [5], [6], [7].

The control system is constituted by both the numerical model of physical system via finite element method and its controller, as it can be seen in Fig. 1. However, the numerical field simulation and the analysis of control system often belong to two different software systems without any strong interactions. One of the possibilities is the integration of the numerical field computation and the control system into one simulation tool in the frame of the Matlab-Simulink system simulator [8]. The controller can be realized in the Matlab/Simulink environment [8], [9], [10]. The field computation parts are implemented under the Matlab computing environment in $\mathrm{C}$ language and in own scripting language of the Matlab. It can be embedded in the Simulink environment easily [10].

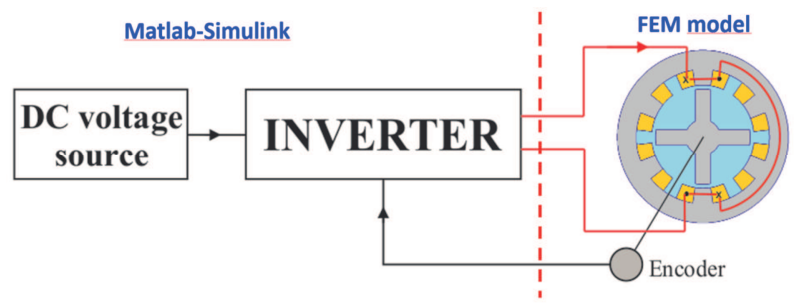

Fig. 1. Block diagram of the switched reluctance drive system

Nevertheless, the computation time of FEM within control loop is far longer than simulating with a state space model, because of the large sparse system of equations. There is an obvious solution to this problem, namely the parallelization of finite element method [6], [7], [11], [12], [13] in order to speed-up the computations. One of the possibilities is the Domain Decomposition Method (DDM) [6], [7], [13], [14], [15], [16], [17]. The used DDM is the Schur-complement method [13], [14], [15] with the parallel preconditioned conjugate gradient method [13], [14], which is currently one of the most popular methods for systems with real symmetric positive definite matrices.

This work presents the closed loop control of a switched reluctance motor, when the parallel finite element model of SRM is used. Additionally, the external circuit and mechanical equations coupled with the finite element method is used to the suitable 
waveform of motor input and output signals. In addition, the performance of the parallel coupled motor model and the control loop are compared with the current excited parallel model. Moreover, the rotor position based control is applied to the FEM model, which seems to be an appropriate control strategy, when the electrical angle of rotor is convenient when considering commutation.

\section{Parallel finite element model of the motor}

The switched reluctance motor has been modelled by the time-stepping parallel finite element method. The FEM formulation relies in a direct coupling between magnetic field, driving circuit and mechanical oscillation equations yielding a complete description of the state of the motor at every time instance during the numerical simulation [3], [5], [6], [7], [9].

\subsection{Field-circuit coupled model of the motor}

The switched reluctance motor has been simulated supposing a two-dimensional problem. The assumption of the two-dimensionality is usually valid if the axial length of the analyzed body is far greater than the dimensions in the cross-sectional plane. In the switched reluctance motor, this is usually not the case. This can be seen in Fig. 2 . However, the magnetic flux flows only through the laminated structure of the rotor and stator, so the two-dimensional assumption is valid.
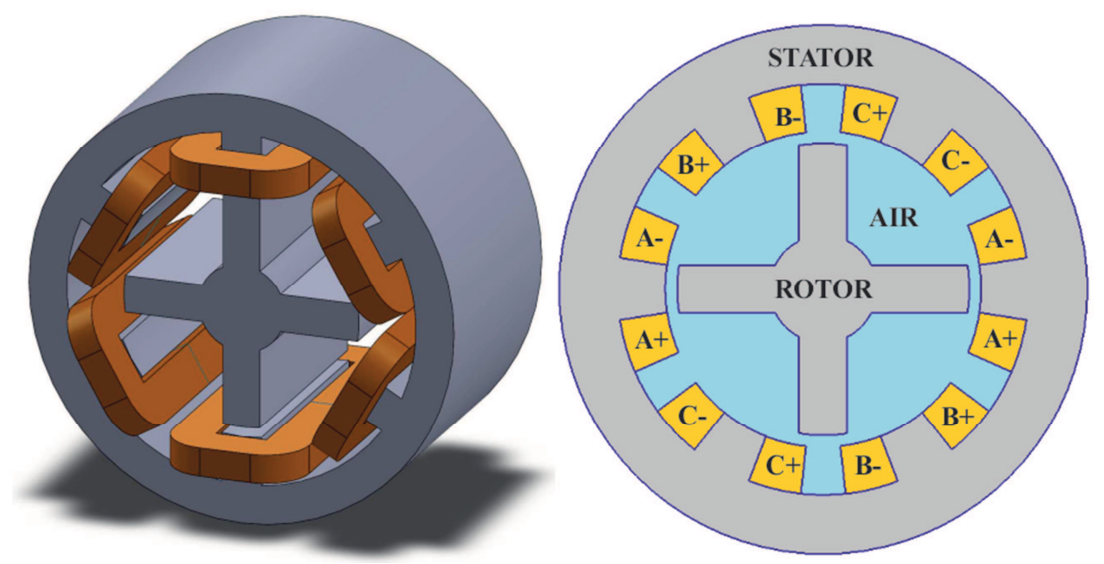

Fig. 2. The structure of a $6 / 4$ switched reluctance motor and the radial cross-section of the motor

In the field model the eddy current effect on the regions of rotor and stator core is taken into account. The electromagnetic field can be expressed in term of a state variable, the magnetic vector potential, $\boldsymbol{A}$ [4], [5]. In this formulation the magnetic flux density can be expressed as the circulation of the magnetic vector potential [4], [5], and $\boldsymbol{B}=\operatorname{curl} \boldsymbol{A}$ has only $z$-component i.e. $\boldsymbol{A}=\left(0,0, A_{\mathrm{z}}\right)$ in Cartesian coordinate system. The 
following well-known equation describes the magnetic field due to the winding currents and eddy currents resulting from transformation of the electromagnetic field [4], [5], [9]

$$
\frac{\partial}{\partial x}\left(\frac{1}{\mu} \frac{\partial A_{z}}{\partial x}\right)+\frac{\partial}{\partial y}\left(\frac{1}{\mu} \frac{\partial A_{z}}{\partial y}\right)-\sigma \frac{\partial A_{z}}{\partial t}=J_{z},
$$

where $\mu$ is the permeability; $\sigma$ is the conductivity and the third term of left-hand side is the induced electric field. The summarization of externally forced currents in the windings is represented by $J_{z}$.

The equation describing the circuit supplying one phase of the switched reluctance motor may be written as [5], [10], [11]

$$
\frac{N_{c} \cdot l_{c}}{S_{c}} \int_{S} \frac{\partial A_{z}}{\partial t} d S+R I_{c}=U_{S}
$$

where $N_{c}$ turns of conductors with cross section $S_{c}$; and $I_{c}$ is the phase current in the conductor; $R$ is the d.c. resistance of a conductor; $U_{s}$ is the supply voltage at the terminal; and $l_{c}$ is the length of the conductor.

The equation system arise from (1) has been solved iteratively by means of a time stepping technique, where the temporal derivatives are discretized by the backward Euler's scheme. To make this possible, the problem domain is needed to be spatially discretized using finite element method, which is based on the weak formulation of the partial differential equations.

In order to facilitate the coupling between the magnetic field equation (1) and circuit equation (2), the phase current density $J_{z}$ can be expressed as

$$
J_{z}=\frac{N_{c}}{S_{c}} I_{c}
$$

The coupled model of the switched reluctance motor can be expressed in matrix notation as [5], [10]

$$
\left[\begin{array}{cc}
\mathbf{S}+\frac{1}{\Delta t} \mathbf{N} & -\mathbf{P} \\
\frac{1}{\Delta t} \mathbf{Q} & \mathbf{R}
\end{array}\right]\left[\begin{array}{l}
\mathbf{A}^{t+\Delta t} \\
\mathbf{I}^{t+\Delta t}
\end{array}\right]=\left[\begin{array}{cc}
\frac{1}{\Delta t} \mathbf{N} & \mathbf{0} \\
\frac{1}{\Delta t} \mathbf{Q} & \mathbf{0}
\end{array}\right]\left[\begin{array}{l}
\mathbf{A}^{t} \\
\mathbf{I}^{t}
\end{array}\right]+\left[\begin{array}{c}
\mathbf{0} \\
\mathbf{U}^{t+\Delta t}
\end{array}\right],
$$

where $\mathbf{A}$ is the vector of magnetic vector potential; $\mathbf{I}$ is the vector of currents in the windings; $\mathbf{U}$ is the vector of voltages at the terminal of the winding; $\mathbf{S}$ is the matrix related to permeability; $\mathbf{N}$ is the matrix related to electric conductivity; $\mathbf{P}$ is the matrix associated with constant coil current; $\mathbf{Q}$ is the matrix associated with flux linkage; $\mathbf{R}$ is the matrix of d.c. resistance of windings. 
The above described formulation is the voltage fed formulation of the motor model. The formulation of current fed model is based on equation (1) and (3).

\subsection{Mechanical motion model}

The mechanical motion of the rotor is a result of electromagnetic torque acting on the rotor. The force can be derived using the Maxwell's stress tensor. To calculate electromagnetic torque the integral is performed using the following equation [5]

$$
\boldsymbol{T}_{e}=l_{z} \int_{\Gamma}\left\{\boldsymbol{r} \times\left[\frac{1}{\mu}(\boldsymbol{B n}) \boldsymbol{B}-\frac{1}{2 \mu_{0}} B^{2} \boldsymbol{n}\right]\right\} d \Gamma,
$$

where $l_{z}$ is the active axial length; $\boldsymbol{r}$ is the position vector linking the rotation axis to the element $\mathrm{d} \Gamma$; and $\Gamma$ is a surface, which is placed in the middle of the air gap; $\boldsymbol{B}$ is the magnetic flux density; $\mu_{0}$ is the permeability of vacuum; and $\boldsymbol{n}$ is the normal unit vector to the surface.

The motion of the switched reluctance motor is analyzed using a well-known second order differential equation [3], [5]

$$
\begin{aligned}
& J_{r} \frac{d \omega}{d t}-D_{r} \omega=T_{e}-T_{L}, \\
& \omega=\frac{d \varphi}{d t},
\end{aligned}
$$

where $J_{r}$ is the rotor inertia moment; $D_{r}$ is the friction damping coefficient; $T_{e}$ is the electromagnetic torque; $T_{L}$ is the load torque acting on the mechanical axis; $\omega$ is the rotor angular velocity; and $\varphi$ is the rotor angular position.

Applying time discretization using Euler's method the following system of equation results [3]

$$
\left[\begin{array}{cc}
1 & -\Delta t \\
0 & 1+\Delta t \frac{D_{r}}{J_{r}} \boldsymbol{R}
\end{array}\right]\left[\begin{array}{l}
\varphi^{t+\Delta t} \\
\omega^{t+\Delta t}
\end{array}\right]=\left[\begin{array}{c}
\varphi^{t} \\
\omega^{t}+\Delta t \frac{1}{J_{r}}\left(T_{e}^{t}-T_{L}\right)
\end{array}\right]
$$

Using a time-stepping method the rotor displacement is calculated at each time step and then transformed into discretized space using the sliding surface technique [16], [17], [18]. Furthermore, the first order interpolation technique has been used [19], which guarantees the continuity, when the stator and rotor part of the mesh are nonconforming because of variation of angular speed. 


\subsection{Parallel coupled FEM model}

When domain decomposition method is used, the problem domain $\Omega$ is divided into several sub-domains in which the unknowns can be calculated simultaneously, i.e. in a parallel way. The general form of a linear algebraic problem arising from the discretization of our problem defined on the domain $\Omega$ can be written as $\mathbf{K a}=\mathbf{b}$, in more detail:

$$
\mathbf{K}=\left[\begin{array}{cc}
\mathbf{S}+\frac{1}{\Delta t} \mathbf{N} & -\mathbf{P} \\
\frac{1}{\Delta t} \mathbf{Q} & \mathbf{R}
\end{array}\right], \quad \mathbf{a}=\left[\begin{array}{l}
\mathbf{A}^{t+\Delta t} \\
\mathbf{I}^{t+\Delta t}
\end{array}\right], \quad \mathbf{b}=\left[\begin{array}{cc}
\frac{1}{\Delta t} \mathbf{N} & \mathbf{0} \\
\frac{1}{\Delta t} \mathbf{Q} & \mathbf{0}
\end{array}\right]\left[\begin{array}{c}
\mathbf{A}^{t} \\
\mathbf{I}^{t}
\end{array}\right]+\left[\begin{array}{c}
\mathbf{0} \\
\mathbf{U}^{t+\Delta t}
\end{array}\right],
$$

where $\mathbf{K} \in R^{(n \times n)}$ is the mass matrix; $\mathbf{b} \in R^{(n \times 1)}$ on the right hand side of the equations; and $\mathbf{a} \in R^{(n \times 1)}$ contains the unknowns. Here $n$ is a number of unknowns.

After the problem is partitioned into a set of $N_{S}$ disconnected substructures, as it can be seen in Fig. 3. The linear sparse system, Ka=b has been split into $N_{S}$ particular blocks [13], [14], [15]. It should be noted, that it is much easier to take into account the mechanical motional equation in the parallel computation, if the sliding surface is used as an artificial interface boundary in the air gap, as it can be seen in Fig. 3. This means, the stator and rotor are partitioned separately, because this is one of the way efficient parallel computation in moving structure [16], [17]. In this case, the efficient load balancing is compromised, but only the connectivity of artificial boundary, the Shurcomplement of the problem is changed in every instant, when the rotor is rotate.

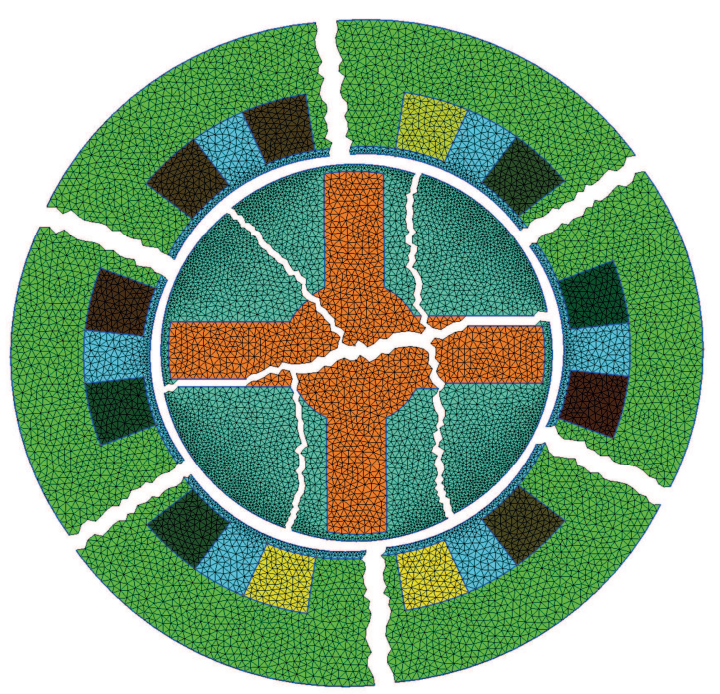

Fig. 3. Twelve substructures of the discretization of switched reluctance motor 
The assembly and the solution of the sub-matrices can be performed parallel by independent processors. However, the solution requires the exchange of interface value, between the processes in charge of the various sub-domains. In many practical applications, the conjugate gradient method is used because of its simplicity and efficiency. Hence, the solution of our problem can be obtained by applying a preconditioned conjugate gradient equation solver with diagonal preconditioning matrix [14]. The parallel implementation of the preconditioned conjugate gradient method can be found in [13], [14].

The geometry has been meshed by the help of first order triangular element generated by Gmsh (a three-dimensional finite element mesh generator with built-in pre- and post-processing facilities) [20]. The used mesh consists of 94389 first-order triangular elements, and the number of unknown potentials is 46048 . The number of unknowns with the unknown currents is 46054 . In Fig. 4 the assembled solution of the problem can be seen.

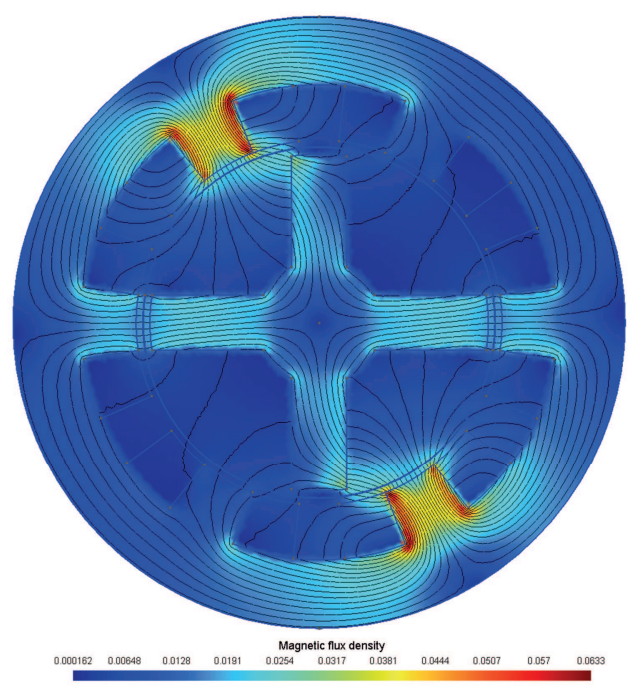

Fig. 4. The surface plot of magnetic flux density and the equipotential lines of magnetic vector potential inside the SRM

\section{Closed loop position control of switched reluctance motor}

To achieve good performances the SRM drive requires an accurate shaft position feedback signal, implying the need for a high quality encoder [1], [21] (as it can be seen in Fig. 1) or, another way, a sophisticated sensor-less controller [1], [2]. The position sensor used in SRM drives have the disadvantages of extra cost, electrical supply, mechanical alignment problems, less suitability to space restricted applications, like vehicle applications and significant disadvantage of being inherent source of unreliability. On the other hand the sensor-less controllers need extra computation time 
and memory to cover the huge amounts of mathematical operations and large lookup tables (e.g. linkage flux and torque networks as a function of current and position) required by the implementation of the advanced control strategy. Therefore they need costly very high performance processors. Due to these drawbacks most of the commercial controllers of SRM drives use sensors [1], [21].

In this case, a simple control strategy used with the field-circuit coupling finite element model of the 6/4 (six stator per four rotor poles) SRM. The position of the rotor was used for controlling the turning on and turning off angles of the excitation after each time step. The speed, current and torque are not used to control the dynamic response of the motor, because the control strategy is not the main focus of the research.

The control loop is implemented in Matlab-Simulink (as it is shown in Fig. 1), where the inverter is not a power electronic circuit, the inverter is a switching block, which work as the asymmetric H-bridge converter [1] and it can excite the appropriate winding based on the rotor position.

The parallel finite element model of SRM is implemented as an interpreted Matlab function, which contains the finite element procedure, which is presented in Section 2. The finite element procedure means loading the finite element mesh from Gmsh, and data of materials, assembling the coupled equation system and solving it. Loading the data load and assembling of the permanent part of equation system have been done only in the first time step.

\section{Numerical experiment}

In numerical experiment the rotor position control of switched reluctance motor is considered. The current excited static magnetic version of this problem is an Agros2D [22] example. The geometry and parameters are from [22].

In this paper the parameters of modified example are the following, the number of turns of the windings is 15 , and the coil resistance is $0.4 \Omega$. The B-H relationship of the iron is considered to be linear, because the magnetic flux density is not so high (as it is shown in Fig. 4), and the motor operates in the linear slope of the magnetization curve. The relative permeability is 1000 , and the conductivity of iron is $1.39 \cdot 10^{6} \mathrm{~S} / \mathrm{m}$. The amplitude of the square wave current excitation is $13.1 \mathrm{~A}$, and square wave voltage excitation is $10 \mathrm{~V}$. The coils are assumed to be symmetrically wound, and eddy current effects are neglected.

The implemented control loop is analyzed with current fed and voltage fed model. The current fed model is useful for validating the correct operation of the implemented parallel program. Fig. 5 shows the computed electromagnetic torque as a function of mechanical angle, and as it can be seen the result of current fed model is almost the same as the current fed results of Agros2D free finite element software. Further, there is a big difference between the results of current and voltage fed FEM models, because the effects of self-inductance and mutual inductance are neglected in the case of current fed models. This conclusion is also supported by Fig. 6. Fig. 6 shows the phase currents as a function of rotor position. The upper figure shows the square waveform current of the current fed model, and the lower figure shows the calculated current waveform of the voltage fed model. 


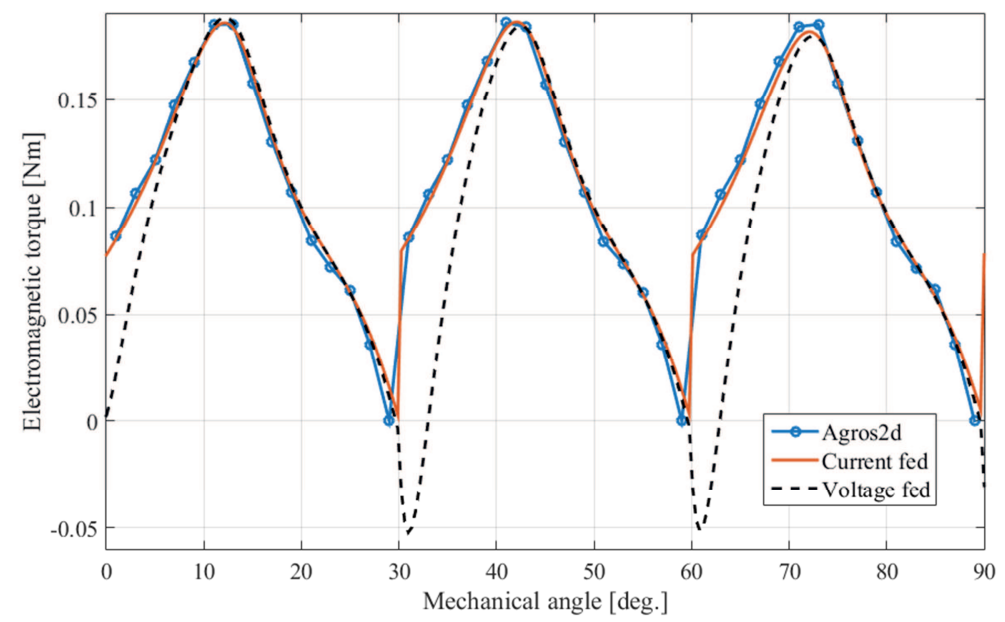

Fig. 5. Electromagnetic torque waveforms obtained the voltage fed model and current fed model
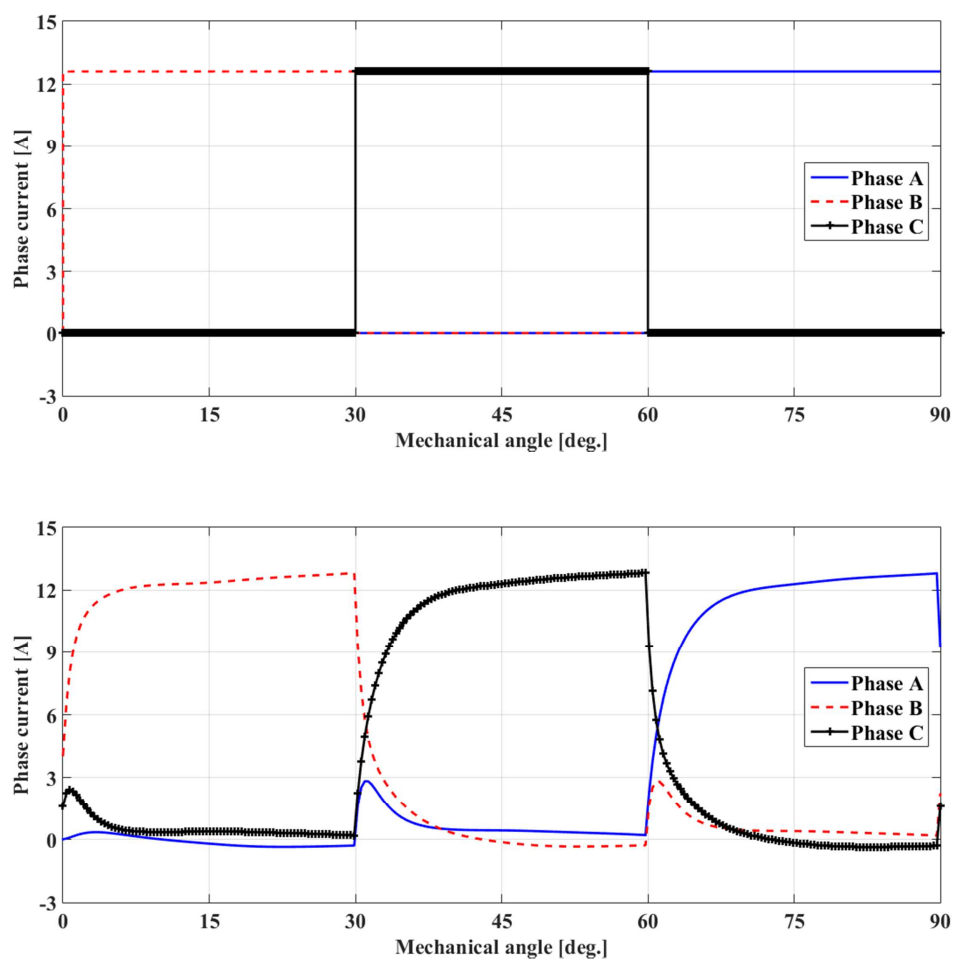

Fig. 6. Phase currents of the current excited model (upper) and voltage excited model (lower) 
The next figure (Fig. 7) shows the start-up of the switched reluctance motor, when the load torque is $0.07 \mathrm{Nm}$. This figure shows more significant difference between the current and voltage fed models than the previous figures. The steady state angular speed of current fed model is approximately three times larger, than the voltage fed, however the amplitude of the current waves is more or less same. Position based control is used for the motor, so the voltage fed model is reached the desired angular position three times slower than the current fed model. Besides, the ripple torque is increased in the case of voltage excitation, because the torque is depend from the inductances and phase current, and the inductances are a function of the rotor position and the phase current. So, the different effects of the switched reluctance machine are hardly depend on each other, and the robustness and performance of the control loop are dependent on the accurate modelling of the machine.

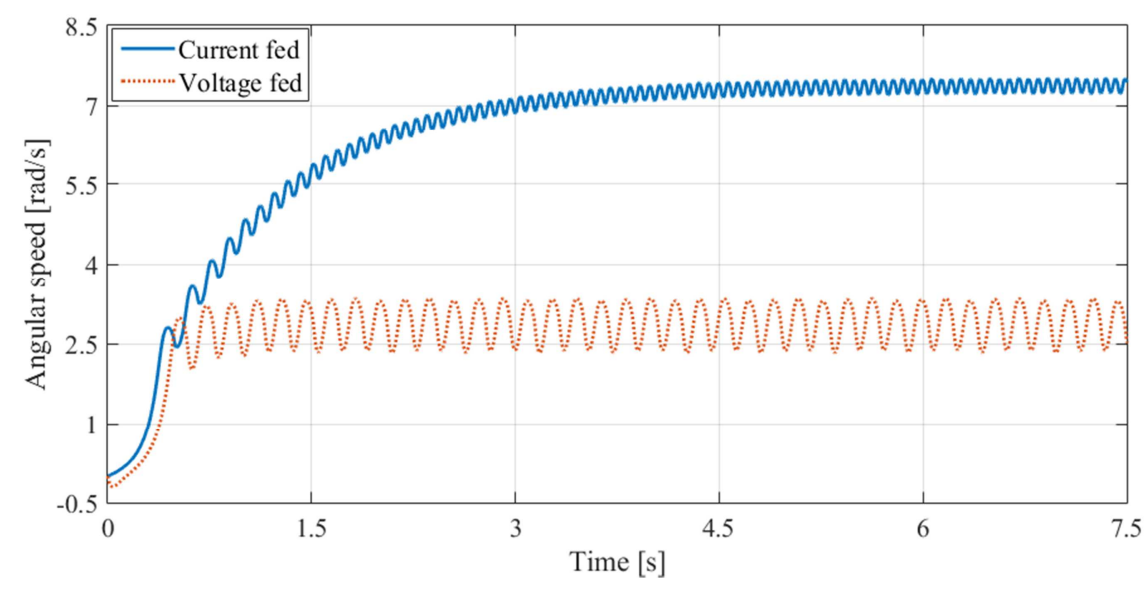

Fig. 7. Speed variation of the motor in time

The parallel performance of the position control of the switched reluctance motor is summarized in Fig. 8. The speedups are computed using the wall clock time of sequential calculation as the reference point. The results show a speedup as high as 2.1 for the control loop with current fed model, and 1.9 for the control loop with voltage fed model. However, the curve of the voltage fed model is continually upward-sloping, when the speedup of current fed model is decreased in the case for 16 sub-domains. In other words, the critical number of sub-domains is more than 16 for the voltage fed model, therefore it is possible to get more speedup in this case.

\section{Conclusion}

This paper presents the field-circuit coupling parallel finite element model embedded in position based closed loop control for analysis of a switched reluctance motor. The behavior of the SRM drive is analyzed by a current excited model and by a 
voltage excited model. The computation time of the numerical model is reduced nearly half of time by domain decomposition method.

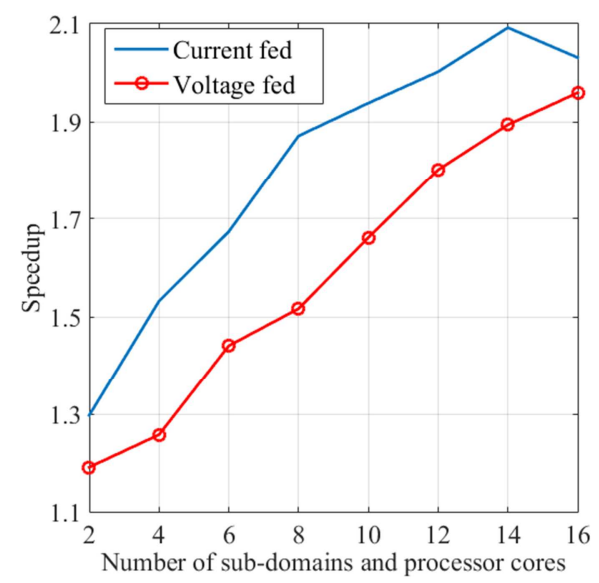

Fig. 8. Performance results for the control loops with parallel FEM

It can be concluded, that a control combining robustness and good performance is depending on an accurate modelling of the machine. The design of drive system requires an appropriate model of the electric machine, without significant simplification, which limit its accuracy. Further, this simple control problem shows the advance of using the finite element model in the control loop in the Matlab-Simulink environment is that the parallel numerical field analysis can be easily interconnected with different control strategies.

The aim of future research is to decrease the computation time of the model by the help of another domain decomposition method, and the torque ripple of the switched reluctance motor by an appropriate control method.

\section{References}

[1] Hilairet M., Lubin T., Tounzi A. Variable reluctance machines, Modeling and control, In Control of Non-conventional Synchronous Motors, Louis J. P. (Ed.) London, Wiley, 2011.

[2] Miller T. J. E. Switched reluctace machines, In Handbook of Electric Motors, Toliyat H. A., Kliman G. B. (Eds.) Boca Raton, FL, CRC Press, 2004.

[3] Bernat J., Stepien S., Stranz A., Szymanski G., Sykulski J. K. Infinte time horizon optimal control of a stepper motor exploiting a finite element model, Bulletin of the Polish Academy of Science, Technical Science, Vol. 62, No. 4, 2014, pp. 835-841.

[4] Kuczmann M., Iványi A. The finite element method in magnetics, Budapest, Akadémiai Kiadó, 2008.

[5] Bastos J. P. A., Sadowski N. Electromagnetic modeling by finite element methods, New York, Marcel Dekker, Inc, 2003. 
[6] Yao W., Jin J. M., Krein P. T. A highly efficient domain decomposition method applied to 3-D finite-element analysis of electromechanical and electric machine problems, IEEE Transactions on Energy Conversion, Vol. 27, No. 4, 2012, pp. 1078-1086.

[7] Yao W., Jin J. M., Krein P. T., Magill M. P. A finite-element-based domain decomposition method for efficient simulation of nonlinear electromechanical problems, IEEE Transactions on Energy Conversion, Vol. 29, No. 2, 2014, pp. 309-319.

[8] Matlab/Simulink, http://www.mathworks.com (Last visited 6 February 2016).

[9] Kanerva S., Simulation of electrical machines, circuits and control systems using finite element method and system simulator, Espoo, Doctoral Thesis, 2005.

[10] Marcsa D., Kuczmann M. Closed loop control of finite element model in magnetic system, Pollack Periodica, Vol. 10, No. 3, 2015, pp. 19-30.

[11] Takahashi Y., Iwashita T., Nakashima H., Tokumasu T., Fujita M., Wakao S., Fujiwara K., Ishihara Y. Parallel time-periodic finite-element method for steady-state analysis for rotating machines, IEEE Transactions on Magnetics, Vol. 48, No. 2, 2012, pp. 1019-1022.

[12] Keränen J., Pippuri J., Malinen M., Ruokolainen J., Råback P., Lyly M., Tammi K. Efficient parallel 3-D computation of electrical machines with elmer, IEEE Transactions on Magnetics, Vol. 51, No. 3, 2015, Article\#: 7203704.

[13] Magoulés F., Roux F. X. Algorithms and theory for sub-structuring and domain decomposition methods, In Mesh Partitioning Techniques and Domain Decomposition Methods, Magoulés F. (Ed.) Kippen, Stirling, Saxe-Coburg Publications, 2007.

[14] Nikishkov G. P. Basics of the domain decomposition method for finite element analysis, In Mesh Partitioning Techniques and Domain Decomposition Methods, Magoulés F. (Ed.) Kippen, Stirling, Saxe-Coburg Publications, 2007.

[15] Marcsa D., Kuczmann M. Performance study of domain decomposition methods for 2D parallel finite element analysis, Pollack Periodica, Vol. 8, No. 3, 2013, pp. 47-58.

[16] Takahashi Y., Fujiwara K., Iwashita T., Nakashima H. Parallel finite-element analysis of rotating machines based on domain decomposition considering nonconforming mesh connection, IEEE Transactions on Magnetics, Vol. 52, No. 3, 2016, Article\#: 7401604.

[17] Böhmer S., Lange E., Hafner M., Cramer T., Bischof C., Hameyer K. Mesh decomposition for efficient parallel computing of electrical machines by mof FEM accounting for motion, IEEE Transactions on Magnetics, Vol. 48, No. 2, 2012, pp. 891-894.

[18] Li H. L., Ho S. L., Fu N. W. Precise magnetic field modeling techniques of rotary machines using transient finite-element method, IEEE Transactions on Magnetics, Vol. 48, No. 11, 2012, pp. 4192-4195.

[19] Perrin-Bir R., Coulomb J. L. A three dimensional finite element mesh connection for problems involving movement, IEEE Transactions on Magnetics, Vol. 31, No. 3, 1995, pp. 1920-1923.

[20] Kuczmann M., Budai T., Kovács G., Marcsa D., Friedl G., Prukner P., Unger T., Tomozi Gy. Application of PETSC and other useful packages in finite element simulation, Pollack Periodica, Vol. 8, No. 2, 2013, pp. 141-148.

[21] Castro J., Andrada O., Blanque B. Minimization of torque ripple in switched reluctance motor drives using direct instantaneous torque control, Proceedings of International Conference in Renewable Energies and Power Quality (ICREPQ'12), Santiago de Compostela, Spain, 28-30 March, 2012, pp. 1-5.

[22] Agros2D, http://www.agros2d.org/ (Last visited 6 February 2016). 